\title{
Knockdown of angiopoietin-like 4 inhibits the development of human gastric cancer
}

\author{
JIN-WU CHEN ${ }^{1 *}$, YING-JIA LUO ${ }^{2 *}$, ZHENG-FEI YANG $^{3}$, LI-QIANG WEN ${ }^{3}$ and LIN HUANG ${ }^{4}$ \\ ${ }^{1}$ Medical Examination Center, and Departments of ${ }^{2}$ Teaching and Research of Diagnostics, ${ }^{3}$ Emergency and \\ ${ }^{4}$ Center for Spine and Pelvic Tumors, Orthopedic Department, Sun Yat-sen Memorial Hospital, \\ Sun Yat-sen University, Guangzhou, Guangdong 510120, P.R. China
}

Received June 15, 2017; Accepted January 2, 2018

DOI: $10.3892 /$ or.2018.6253

\begin{abstract}
Human gastric cancer (GC) is the second most common cause of cancer-related deaths worldwide and is one of the most common metastatic cancers. Tumor proliferation, apoptosis, metastasis and invasion are important predictors of the invasiveness of GC and are key factors in cancer-induced death. Angiopoietin-like 4 (ANGPTL4) is a secreted protein that belongs to the angiopoietin (ANGPTL) family and is involved in the regulation of cancer metastasis. However, whether ANGPTL4 plays a role in the progression of GC remain unclear. In the present study, immunoreactivity of ANGPTL4 demonstrated that ANGPTL4 expression was upregulated in GC tissues with the development of GC. The siRNA targeting ANGPTL4 effectively knocked down ANGPTL4 in the SNU-1 and BGC823 cell lines at the mRNA and protein levels. Following ANGPTL4 downregulation, the proliferation and invasion abilities of GC cell lines were suppressed as determined by MTT and Transwell assays, and cell apoptosis level and sensitivity to cisplatin were increased as determined by flow cytometry and MTT assay. In conclusion, these findings suggest that ANGPTL4 may be a new potential therapeutic target for GC.
\end{abstract}

\section{Introduction}

Despite the apparent decline in the incidence of GC in the last few decades, GC currently remains one of the most common cancers worldwide (1). It was reported that the GC mortality

Correspondence to: Dr Li-Qiang Wen, Department of Emergency, Sun Yat-sen Memorial Hospital, Sun Yat-sen University, 107 Yanjiang West Road, Guangzhou, Guangdong 510120, P.R. China

E-mail:wenlq@mail.sysu.edu.cn

Dr Lin Huang, Department of Orthopedic, Sun Yat-sen Memorial Hospital, Sun Yat-sen University, 107 Yanjiang West Road, Guangzhou, Guangdong 510120, P.R. China

E-mail: drhuanglin@139.com

*Joint first authorship

Key words: angiopoietin-like 4, gastric cancer, proliferation, metastasis, invasion, cisplatin rate in East Asia countries is $28.1 \%$ for men and $13.0 \%$ for women (2). At present, the major therapeutic strategy of GC includes surgery combined with adjuvant chemotherapy, and evidence suggests that this approach is effective for early GC (3), However, the efficacy of these measures on patients with advanced GC is still unsatisfactory. Due to the lack of effective early diagnostic biomarkers, most patients are diagnosed at a late clinical stage with extremely poor prognosis $(2,4)$. As known, diet and family inheritance are two risk factors that contribute to the occurrence of GC, but chronic gastritis caused by Helicobacter pylori is the primary cause (5-7). In spite of the extraordinary progress achieved in the prevention and treatment of GC, issues remain in terms of the exact underlying mechanism. Thus, elucidating the molecular mechanism of GC is of significant importance for exploring new effective therapies.

It was demonstrated the angiopoietin (ANGPTL) family is associated with many physiological processes, including lipid metabolism (8), cell activity regulation (9) and tumor growth (10). The angiopoietin family consists of 7 ANGPTL proteins, named ANGPTL 1-7. As identified in systemic circulation, their amino acid sequences are slightly conserved. Angiopoietin-like 4 (ANGPTL4), widely expressed in different human organs, contains a C-terminal fibrinogenlike domain and an N-terminal coiled-coil fragment (11-13). Previous reports have revealed the ANGPTL4 is involved in the progression of several human tumors, such as colorectal cancer $(14,15)$, prostate cancer (16), and breast cancer (13). However, it remains unclear whether ANGPTL4 is associated with the progression of GC, and there is an urgent need to explore the basic mechanisms by which they participate and discover new therapies for GC patients.

In the present study, we firstly analyzed the expression of ANGPTL4 in GC tissues. In addition, we generated two effective siRNAs of ANGPTL4, named siRNA1 and siRNA2. We used siRNA1 or siRNA2 to transfect human GC cell lines to block the expression of ANGPTL4. We found that knockdown of ANGPTL4 by siRNAs inhibited cell proliferation, migration and invasion, and promoted apoptosis in both SNU-1 and BGC823 cell lines. In addition, we also found that knockdown of ANGPTL4 effectively increased the cisplatin-induced apoptosis in both SNU-1 and BGC823 cells in a dose-dependent manner. These results demonstrated that ANGPTL4 plays a 
critical role in the progression of $\mathrm{GC}$, and it may provide a new potential target for the treatment of GC.

\section{Materials and methods}

Patients and tissue samples. A total of $40 \mathrm{GC}$ patients were recruited at the Sun Yat-sen Memorial Hospital of Sun Yat-sen University (Guangzhou, Guangdong, China), and tumor and corresponding non-tumor control tissues were collected. After washing with sterile phosphate-buffered saline (PBS), all tissue samples were immediately stored at $-80^{\circ} \mathrm{C}$ until further study. This study was approved by the Ethics Committee of Sun Yat-sen Memorial Hospital, and written informed consent was obtained from all patients.

Cell lines and culture. The GC cell line SNU-1 was obtained from the American Type Culture Collection (ATCC, Manassas, VA, USA), and GC cell line BGC823 was purchased from Shanghai Institutes for Biological Sciences, Chinese Academy of Sciences. Both SNU-1 and BGC823 cells were maintained in RPMI-1640 medium with $100 \mathrm{U} / \mathrm{ml}$ penicillin, streptomycin and $10 \%$ fetal bovine serum (FBS, cat. no. 100-106; Gemini Bio Products, West Sacramento, CA, USA) at $37^{\circ} \mathrm{C}$ and $5 \% \mathrm{CO}_{2}$.

Knockdown of human ANGPTL4 in GC cell lines. Negative control siRNA (NC) and two siRNAs against human ANGPTL4 (siRNA1, siRNA2) were designed and obtained from GenePharma (Shanghai, China). The sequence of siRNA1 was 5'-GGUGACUCUUGGCUCUGCC-3' (sense) and 5'-GGUGACUCUUGGCUCUGCC-3' (antisense). The sequence of siRNA2 was 5'-AGGGAAUCUUCUGGAA GAC-3' (sense) and 5'-GUCUUCCAGAAGAUUCCCU-3' (antisense). According to the manufacturer's instructions, SNU-1 or BGC823 cells were transfected with $40 \mathrm{nmol} / 1$ of ANGPTL4 siRNA1, siRNA2 or NC siRNA using Lipofectamine ${ }^{\circledR} 3000$ (Invitrogen, Carlsbad, CA, USA). qRT-PCR and western blot analysis were used to detect the knockdown efficacy of the ANGPTL4 siRNAs.

RNA extraction and quantitative real-time PCR ( $q R T-P C R)$. Total RNA was extracted from the SNU-1 and BGC823 cells using TRIzol reagent (Invitrogen), and $5 \mu \mathrm{g}$ RNA was used for synthesizing cDNA using the Revert Aid First Strand cDNA Synthesis kit (Invitrogen, UK) according to the manufacturer's instructions. Quantitative real-time PCR was performed using a 7900 HT Fast system (Applied Biosystems, Life Technologies, Foster City, CA, USA). During the course of the reaction, the $\mathrm{Cq}$ value was obtained, and the results were analyzed using $2^{-\Delta \Delta \mathrm{Cq}}$ calculation. Glyceraldehyde 3-phosphate dehydrogenase (GAPDH) was used as an endogenous housekeeping gene. The sequence of human ANGPTL4 PCR primers was 5'-GG CGA GTT CTG GCT GGG TCT-3' (sense) and 5'-TGG CCG TTG AGG TTG GAA TG-3' (antisense). The sequences of the GAPDH primers were: 5'-ATG TCG TGG AGT CTA CTG GC-3' (forward) and 5'-TGA CCT TGC CCA CAG CCT TG-3' (reverse).

Western blot analysis. Total proteins of the human GC cells were prepared using RIPA buffer (cat. no. R3792). Cell lysis was preformed on ice using ultrasonication. Cell debris was centrifuged $\left(13,000 \mathrm{rmp}, 20 \mathrm{~min}, 4^{\circ} \mathrm{C}\right)$ and the supernatant was collected. The concentrations of proteins in each sample were detected using a protein assay reagent (Bio-Rad Laboratories, Inc., Hercules, CA, USA). SDS-PAGE (10\%) was used to separate total proteins $(40 \mu \mathrm{g})$. The separated proteins were then transferred onto Immun-Blot ${ }^{\circledR}$ PVDF membranes (cat. no. 162-0177; Bio-Rad Laboratories). The membranes were blocked with 5\% skim milk in TBS for $2 \mathrm{~h}$ at room temperature, and incubated with the primary antibody overnight at $4^{\circ} \mathrm{C}$. Then the membranes were incubated with secondary horseradish peroxidase antibody (HRP, cat. no. 074-1806; KPL Co., USA). The results were analyzed using a chemiluminescence substrate kit and an ECL system (both from Amersham Pharmacia Biotech Inc., Piscataway, NJ, USA). The primary antibodies were ANGPTL4 (1:1,000; AF3485; R\&D Systems Inc., Minneapolis, MA, USA) and GAPDH (1:200; sc-51631; Santa Cruz Biotechnology, Inc., Santa Cruz, CA, USA).

Cell migration and invasion assays. Cell migration and cell invasion assays were performed using Transwell chambers (8- $\mu \mathrm{m}$ pore size; BD Biosciences, San Jose, CA, USA) with or without Matrigel coating (BD Biosciences). Briefly, SNU-1 or BGC 823 cells $\left(0.2 \mathrm{ml}, 2 \times 10^{5}\right.$ for the invasion assay; $1 \times 10^{5}$ for the migration assay) were seeded in the upper chamber, and the lower chamber was filled with $10 \%$ FBS containing medium $(0.6 \mathrm{ml})$. After $24 \mathrm{~h}$, cells on the upper chamber were removed, and migrated cells were fixed and stained with 5\% crystal violet. The cells were captured and counted under a microscope.

Cell viability assay (MTT assay). After transfection, SNU-1 or BGC823 cells were seeded into a 96-well plate at a concentration of $2 \times 10^{4}$ cells per well. Incubated for $24 \mathrm{~h}$, cells were then washed with PBS and $20 \mu 1$ of MTT solution was added to each well. After at least $30 \mathrm{~min}$ of incubation at $37^{\circ} \mathrm{C}, 150 \mu \mathrm{l}$ dimethyl sulfoxide (DMSO) was added to each well. The plate was then shaken at room temperature for $\leq 10 \mathrm{~min}$, and absorbance was determined at $490 \mathrm{~nm}$ by a microplate reader (Sunrise $^{\mathrm{TM}}$; Tecan Group Ltd., Switzerland).

Apoptosis analysis. Apoptosis rate of GC cells after the transfection of ANGPTL4 siRNA was measured by flow cytometry using Annexin V/PI double staining (BD Biosciences, Franklin Lakes, NJ, USA). The cells were seeded in a 24-well plate at a concentration of $2 \times 10^{4}$ per well. Following overnight incubation, cells were washed at least twice with cold phosphate-buffered saline (PBS). Cells were re-suspended with $0.5 \mathrm{ml}$ binding buffer supplemented with $5 \mu \mathrm{l} \mathrm{PI}$ and $5 \mu \mathrm{l}$ FITC-labeled Annexin V and incubated at room temperature for $10 \mathrm{~min}$ (protected from light). Apoptotic SNU-1 or BGC823 cells were analyzed in triplicates and the analysis was repeated three times independently by flow cytometry on a FACScan (Beckman Instruments, Fullerton, CA, USA).

Immunohistochemical (IHC) staining. Paraffin-embedded tissues were sliced into $4-\mu \mathrm{m}$ sections, and then the sections were treated with xylene and ethanol to remove paraffin, and were rehydrated in PBS. All sections were immersed in $0.01 \mathrm{M}$ sodium citrate buffer $(\mathrm{pH} 6.0)$ at $95^{\circ} \mathrm{C}$ for $\leq 20 \mathrm{~min}$ 
A GC

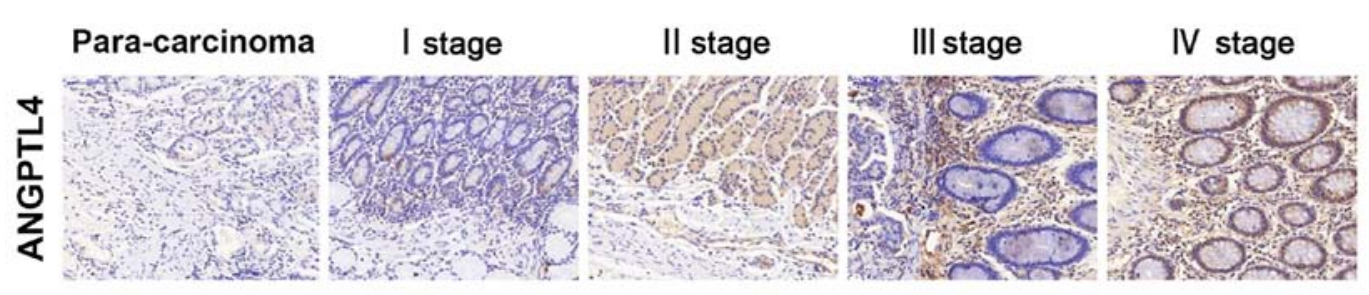

B

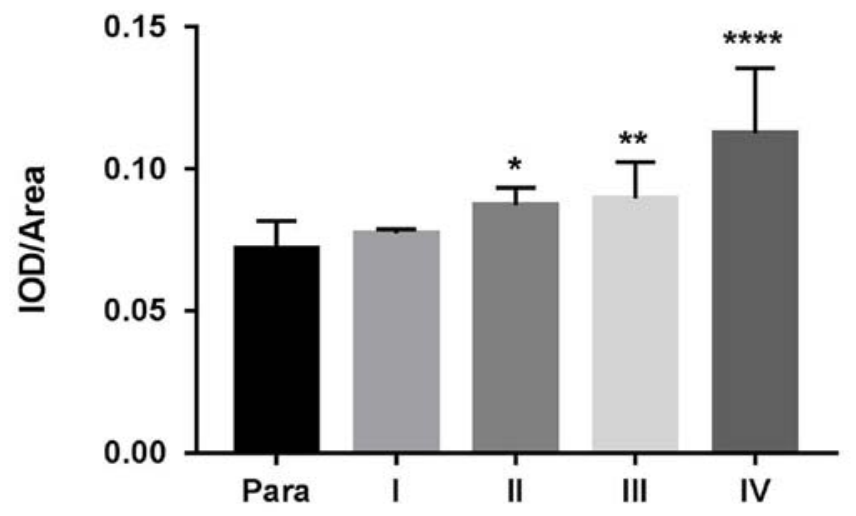

Figure 1. Upregulation of ANGPTL4 in GC tissues. (A) Immunohistochemistry staining was performed to detect the expression level of ANGPTL4 in tumor tissues and adjacent normal tissues from 40 patients with GC (I, II, III and IV stage). (B) Statistical results of the IOD/area. ${ }^{*} \mathrm{P}<0.05,{ }^{* * *} \mathrm{P}<0.01,{ }^{* * * *} \mathrm{P}<0.001$ compared with the para-carcinoma (para) group. ANGPTL4, angiopoietin-like 4. GC, gastric cancer.

for antigen retrieval. The sections were then treated with $3 \%$ $\mathrm{H}_{2} \mathrm{O}_{2}$ for 10 min to block the endogenous peroxidase activity, and incubated with 5\% normal goat serum to block the nonspecific binding. Samples were then incubated overnight at $4^{\circ} \mathrm{C}$ with primary rabbit antibody against human ANGPTL4 (R\&D Systems). After washing with PBS, the sections were sequentially incubated with goat anti-rabbit HRP, and the reaction signals were visualized by the diaminobenzidine reaction (DAB; Dako Ltd.) and sections were counterstained with hematoxylin.

Cisplatin cytotoxicityassay. After transfection with ANGPTL4 siRNA, SNU-1 or BGC823 cells $\left(2 \times 10^{4}\right)$ were seeded in a 96-well plate and incubated overnight at $37^{\circ} \mathrm{C}$. Then cells were treated with various concentrations $(0,2.5,5.0,10.0,20.0$ and $40.0 \mu \mathrm{g} / \mathrm{ml}$ ) of cisplatin (Sigma, Santa Clara, CA, USA). MTT assay was applied to examine cell viability.

Statistical analysis. Statistical analyses were conducted using SPSS 19.0 software (SPSS, Inc., Chicago, IL, USA). All data are expressed as the mean \pm standard deviation (SD). The Student's t-test was carried out to compare the level of cell proliferation, apoptosis, migration, invasion and cisplatin resistance among groups, and $\mathrm{P}<0.05$ was considered to be indicative of statistical significance.

\section{Results}

ANGPTL4 expression is upregulated in GC tissues. To study the roles of ANGPTL4 in GC pathogenesis, protein expression of ANGPLT4 was analyzed by IHC in tumor samples and adjacent normal specimens of 40 patients with GC (I,
II, III and IV stage). The results indicated that the expression of ANGPTL4 was significantly increased in the GC tissues compared with that noted in the normal tissues. In addition, ANGPTL4 expression levels were associated with the clinical phase of GC (Fig. 1).

The expression level of ANGPTL4 is downregulated by siRNA in human GC cell lines. To investigate the role of ANGPTL4 in human GC, two siRNAs targeting ANGPTL4 were designed, and SNU-1 and BGC823 cells were transfected. The knockdown efficiency of those two siRNAs was validated by detecting the mRNA expression level using qRT-PCR assay. Results from qRT-PCR showed that, compared to NC, the mRNA expression of ANGPTL4 in SNU-1 and BGC823 cells was extremely decreased after siRNA1 and siRNA2 transfection $(\mathrm{P}<0.01$ and $\mathrm{P}<0.001$, respectively; Fig. $2 \mathrm{~A}$ and $\mathrm{B})$. We further assessed the ANGPTL4 protein level in the transfected SNU-1 and BGC823 cells, and the results indicated that ANGPTL4 protein was also downregulated (Fig. 2C and D). These results demonstrated that the siRNAs were effective in silencing ANGPTL4 expression.

ANGPTL4 knockdown inhibits cell proliferation and promotes cell apoptosis in GC cell lines. In order to explore the biological function of ANGPTL4 in human GC, we silenced the ANGPTL4 expression by siRNA in SNU-1 and BGC823 cells. Cell proliferation and apoptosis were then detected. MTT assay indicated that knockdown of ANGPTL4 significantly inhibited the cell proliferation in both SNU-1 and BGC823 cells, compared with $\mathrm{NC}(\mathrm{P}<0.05, \mathrm{P}<0.01$ and $\mathrm{P}<0.001$, respecively; Fig. 3). Annexin V/PI staining and flow cytometry assay were employed to further determine whether 
A

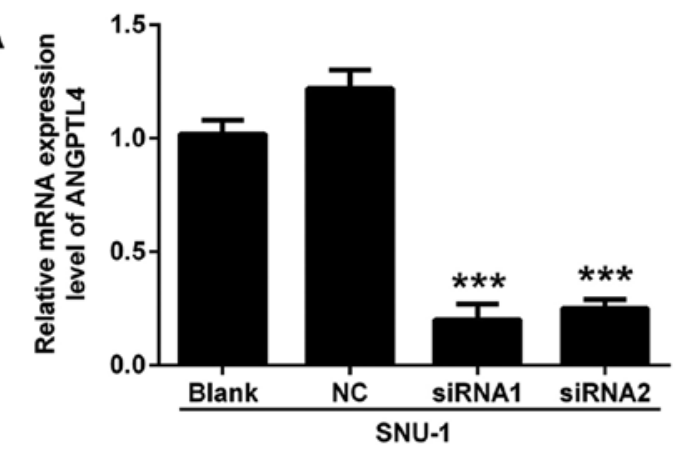

C

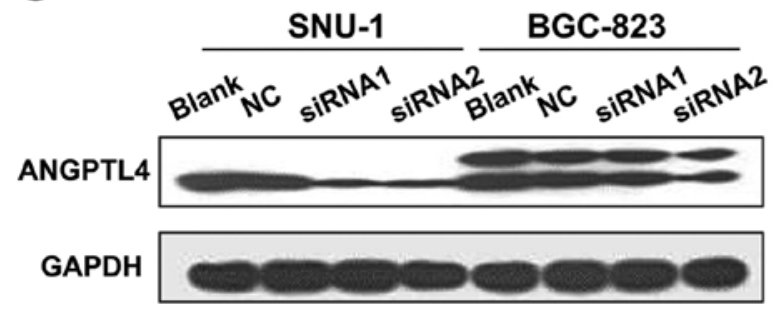

B

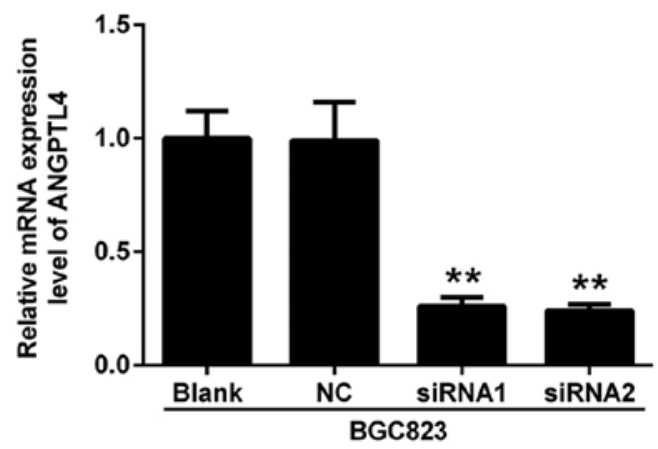

D

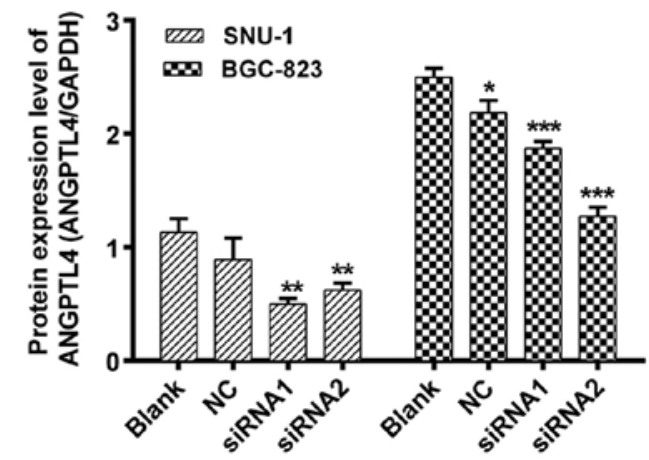

Figure 2. Validation of the knockdown efficiency of two ANGPTL4 siRNAs. SNU-1 and BGC823 cells were transfected with NC, siRNA1 or siRNA2, and the knockdown efficiency of ANGPTL4 was validated by qRT-PCR (A and B) and western blot analysis $(\mathrm{C}$ and $\mathrm{D})$. $^{*} \mathrm{P}<0.05,{ }^{* *} \mathrm{P}<0.01$ or ${ }^{* * * *} \mathrm{P}<0.001$ compared with the negative control (NC) group. ANGPTL4, angiopoietin-like 4.
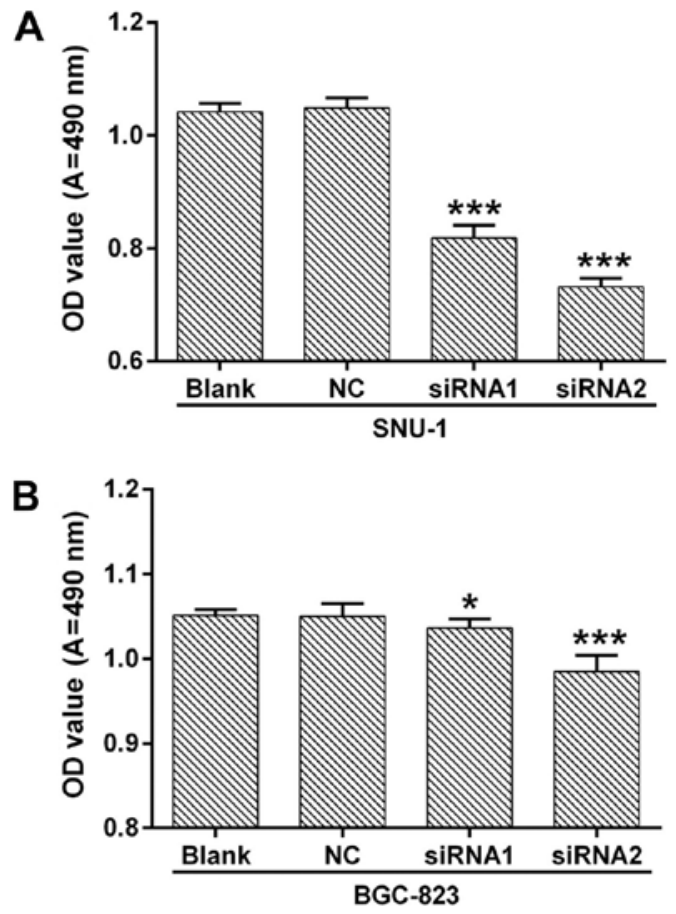

Figure 3. Effects of ANGPTL4 knockdown on human gastric cancer cell proliferation. MTT assay was performed to measure the cell proliferation after the knockdown of ANGPTL4 in SNU-1 (A) and BGC823 cells (B). ${ }^{*} \mathrm{P}<0.05$, ${ }^{* * * *} \mathrm{P}<0.001$ compared with the NC group. ANGPTL4, angiopoietin-like 4. A, absorbance.

cell apoptosis could be affected by ANGPTL4. We found that the percentage of apoptosis was significantly upregulated after the silencing of ANGPTL4 in both the SNU-1 $(\mathrm{P}<0.001$, Fig. 4A) and BGC823 cells ( $\mathrm{P}<0.001$, Fig. 4B). This indicated that knockdown of ANGPTL4 inhibited cell proliferation and promoted cell apoptosis in human GC cell lines.

ANGPTL4 knockdown suppresses the ability of cell migration and invasion in human $G C$ cell lines. Migration and invasion are two important factors involved in the progression of cancer cells. To determine whether ANGPTL4 is involved in the migration and invasion of human GC cells, we performed Transwell assays. The results showed that cell migration and invasion were significantly reduced after transfection of SNU-1 and BGC823 cells by siRNA1 or siRNA2 compared to the blank control $(\mathrm{P}<0.001$, Fig. 5). These results confirm that knockdown of ANGPTL4 inhibited human GC cell migration and invasion.

Knockdown of ANGPTL4 enhances the in vitro sensitivity of human GC cells to cisplatin. Whether the expression of ANGPTL4 affects the sensitivity of human GC cells to cisplatin remains unexplored. To ascertain the effects, after transfection with ANGPTL4 siRNA and stabilization, human GC cells were treated with various concentrations $(0$, $2.5,5.0,10.0,20.0$ and $40.0 \mu \mathrm{g} / \mathrm{ml}$ ) of cisplatin for $24 \mathrm{~h}$. The results of MTT assay revealed that knockdown of ANGPTL4 significantly decreased the viability of the SNU-1 $(\mathrm{P}<0.05$, $\mathrm{P}<0.01, \mathrm{P}<0.001$, Fig. 6A) and BGC823 cells $(\mathrm{P}<0.05, \mathrm{P}<0.01$, $\mathrm{P}<0.001$, Fig. 6B) in response to cisplatin in a dose-dependent manner. These results obviously indicated that ANGPTL4 knockdown enhanced the sensitivity of human GC cells to cisplatin. 
A

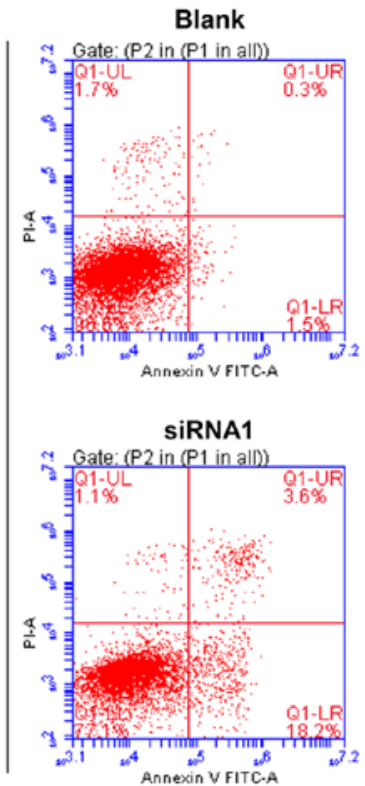

B

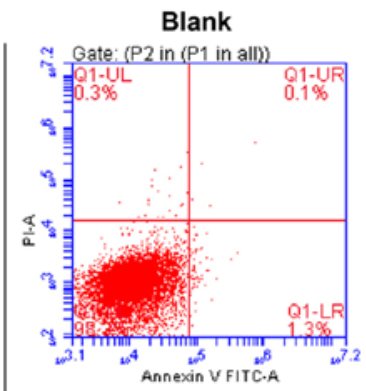

BGC823

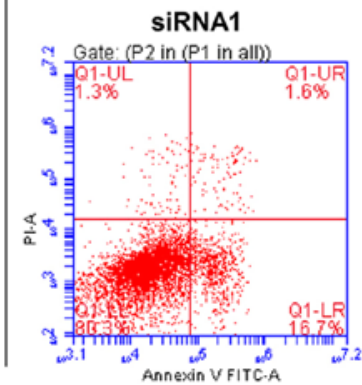

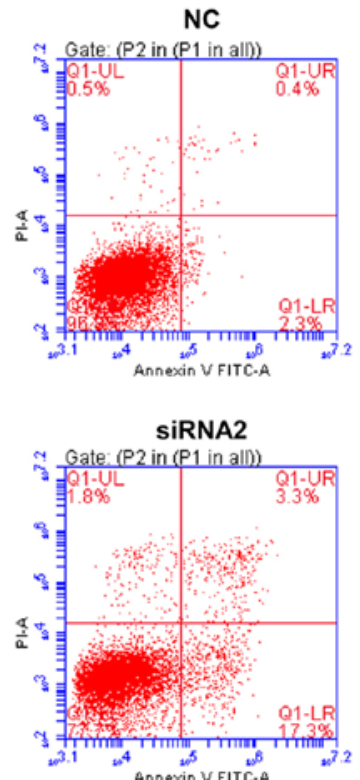

NC

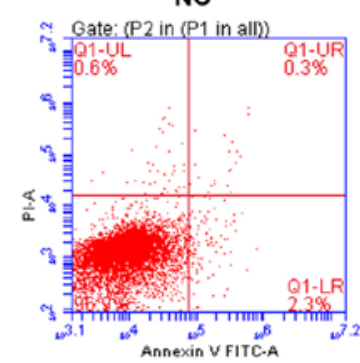

SIRNA2

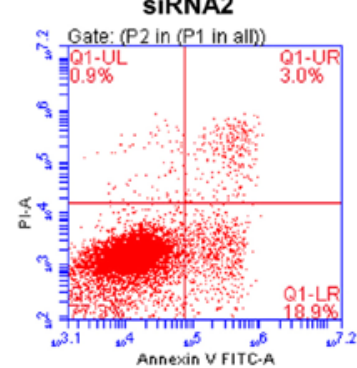

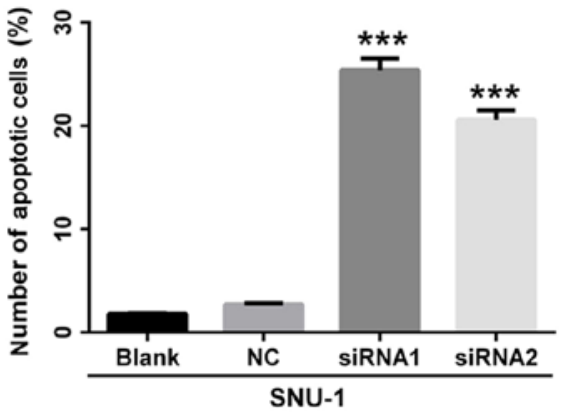

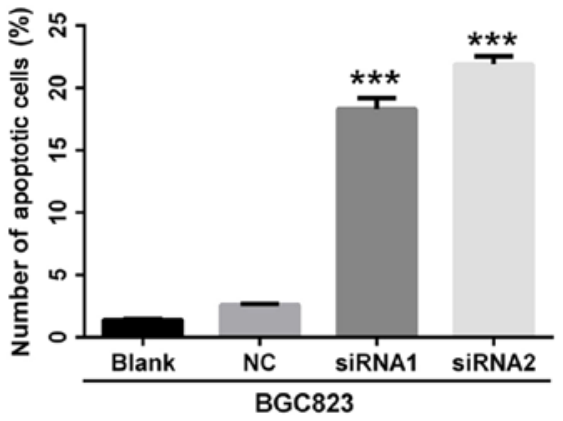

Figure 4. Effects of ANGPTL4 knockdown on human gastric cell apoptosis. Annexin V/PI staining and flow cytometry were carried out to determine the apoptosis rate after siRNA1, siRNA2 or NC were transfected into SNU-1 (A) and BGC823 cells (B), respectively. ${ }^{* * *}$ P $<0.001$ compared with the NC group. ANGPTL4, angiopoietin-like 4.

\section{Discussion}

The poor prognosis of gastric cancer (GC) is mainly due to the lack of early diagnostic biomarkers and effective treatments. Since the prognosis of GC is closely associated with the clinical stage of disease at diagnosis, there is an urgent need to explore novel early diagnostic markers and new effective GC therapies (17).

The communication between tumor cells and their microenvironment is extremely important, as it can significantly affect the efficacy of antitumor treatments $(13,18)$. Matricellular proteins are a type of extracellular matrix (ECM)-associated glycoproteins which are secreted by various tumor cells into the extracellular matrix $(19,20)$. Increasing evidence has shown that several matricellular proteins are involved in the development of various tumor cells, including breast, colorectal and prostate cancer $(15,16,21-24)$. The ANGPTL family is an important novel member of the matricellular proteins and was reported to be involved in many metastatic cancers, indicating that it may play a critical role in the processes of tumor cell metastasis. Among the ANGPTL family, ANGPTL2 has been reported to act as an agent in tumor progression and metastasis in various cancers, such as lung cancer, ovarian cancer and GC (25-27), and may be a key factor in cancer progression. In addition, emerging evidence has shown that ANGPTL4 is upregulated in many human epithelial tumors and is involved in cell migration and prolifration. Zhu et al found that ANGPTL4 overexpression is widespread in tumors, 
A

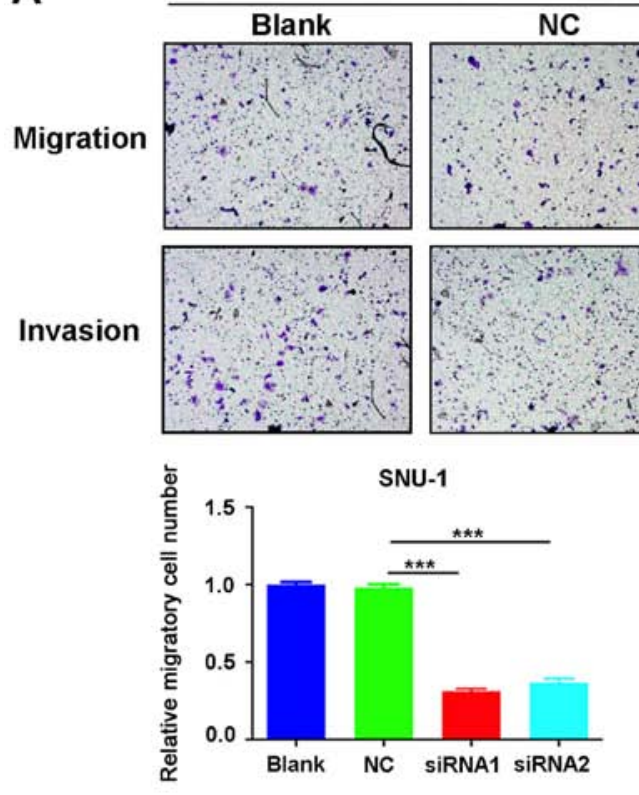

SNU-1

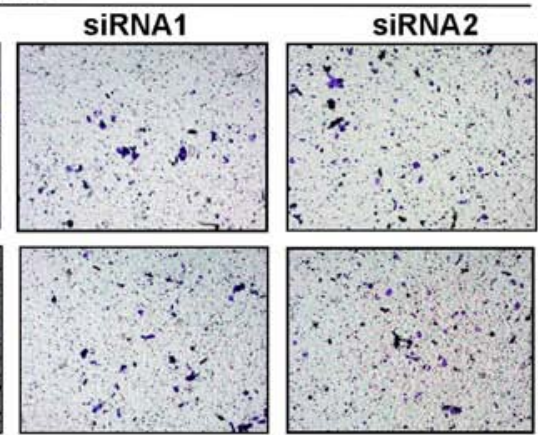

B
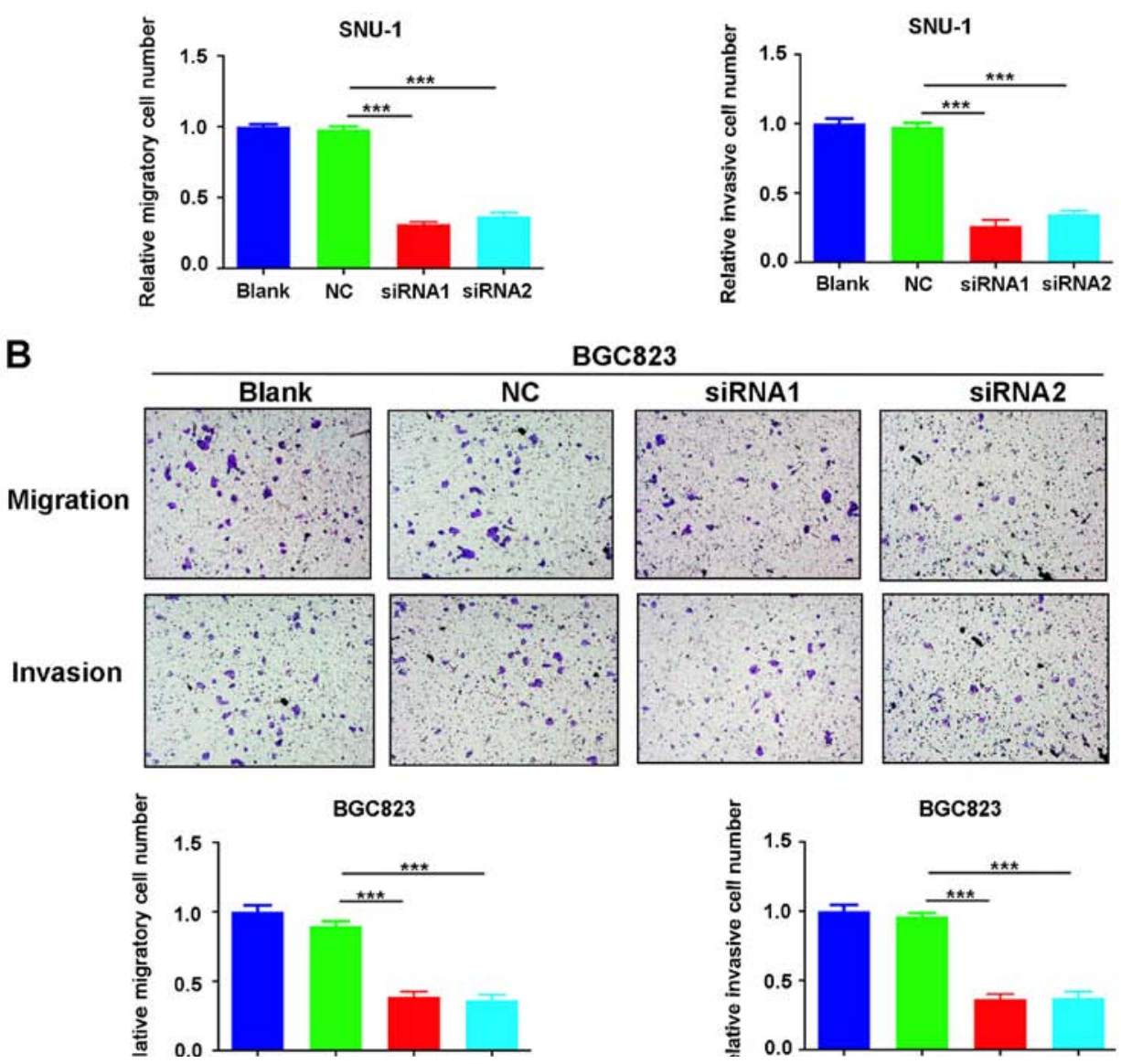

Figure 5. Effects of ANGPTL4 knockdown on migration and invasion ability of human gastric cancer cells. Cell migration and invasion ability was determined by Transwell assays after siRNA1, siRNA2 or NC were transfected into SNU-1 (A) and BGC823 cells (B), respectively. ${ }^{* * *} \mathrm{P}<0.001$ compared with NC groups. ANGPTL4, angiopoietin-like 4.
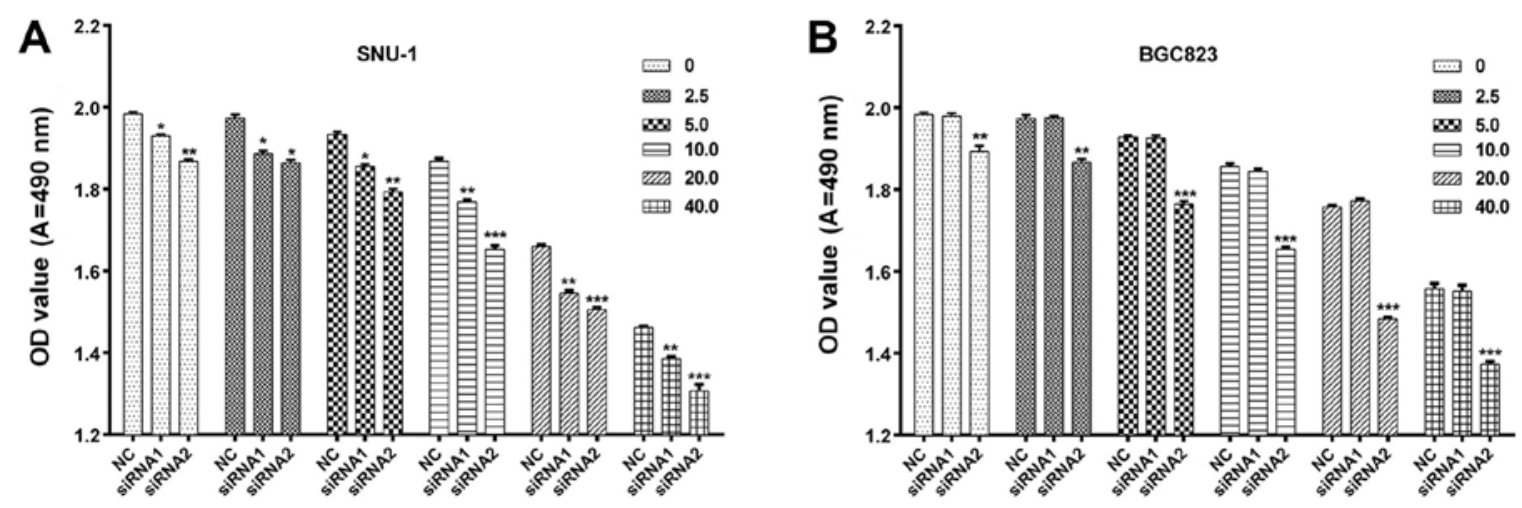

Figure 6. Effects of different concentrations of cisplatin on ANGPTL4-knockdown human gastric cancer cells. MTT assay was used to measure the effects of cisplatin at various concentrations on SNU-1 (A) and BGC823 cells (B) transfected with siRNA1, siRNA2 or NC, respectively $\left({ }^{*} \mathrm{P}<0.05,{ }^{* *} \mathrm{P}<0.01,{ }^{* * * *} \mathrm{P}<0.001\right)$. ANGPTL4, angiopoietin-like 4. 
and promoted tumor growth by elevating the $\mathrm{O}_{2}^{-}: \mathrm{H}_{2} \mathrm{O}_{2}$ ratio (22). Nakayama et al examined the mRNA and protein expression level of ANGPTL4 in 144 human colorectal cancer cases. Their findings suggested that ANGPTL4 is involved in the process of invasion and metastasis (15). Huang et al reported that ANGPTL4 promotes lung cancer metastasis by modulating vascular junction integtity (28). However, the involvement of ANGPTL4 in human GC is unclear and needs further investigation.

In the present study, we found that ANGPTL4 expression was upregulated in GC tissues. In order to further determine the biological functions of ANGPTL4 in human GC, we used siRNA to block the expression of ANGPTL4 in two human GC cell lines, SNU-1 and BGC823, and validated the knockdown efficiency at the mRNA and protein levels by qRT-PCR, and western blot analysis, respectively. MTT and Transwell assays, Annexin V/PI staining and flow cytometry were used to assess the effects of ANGPTL4 knockdown on the GC cells, and results from these assays indicated that knockdown of ANGPTL4 inhibited proliferation, metastasis, invasion and promoted apoptosis in both SNU-1 and BGC823 cell lines.

Surgery and chemotherapy are two main curative treatments for patients with human GC. However, surgery is only effective for patients with early or locally advanced GC (29). Since most patients have advanced or metastatic GC at the time of diagnosis, the efficacy of chemotherapy is limited $(30,31)$. To date, cisplatin remains the most widely used chemotherapeutic agent for advanced GC patients worldwide, although it has several severe side effects, such as asthenia, gastrointestinal disorders and long-term neurological consequences (32). One study demonstrated that ANGPTL4 increased the survival of melanoma cells against cisplatin-induced apoptosis (33). Whether ANGPTL4 affects the efficacy of cisplatin in human GC remains unknown. To explore this, we knocked down ANGPTL4 in SNU-1 and BGC823 cells with siRNA1 or siRNA2 and treated the cells with different concentrations of cisplatin. The results showed that the silencing of ANGPTL4 obviously enhanced the sensitivity of the SNU-1 and BGC823 cells to cisplatin in a dose-dependent manner.

In conclusion, we found that ANGPTL4 participated in GC cell proliferation, apoptosis, and metastasis in vitro, suggesting that it might act as a new potential therapeutic target for GC patients. However, the roles of ANGPTL4 in vivo warrant further investigation.

\section{Acknowledgements}

The present study was supported by a research grant from a project by the Leading Talents in Pearl River Talent Plan of Guangdong Province (no. 81000-42020004) and The Natural Science Foundation of Guangdong (2017A030313652).

\section{References}

1. Zhang XY and Zhang PY: Gastric cancer: Somatic genetics as a guide to therapy. J Med Genet 54: 305-312, 2017.

2. Li H, Yu B, Li J, Su L, Yan M, Zhu Z and Liu B: Overexpression of lncRNA H19 enhances carcinogenesis and metastasis of gastric cancer. Oncotarget 5: 2318-2329, 2014.

3. Li Z, Lei H, Luo M, Wang Y, Dong L, Ma Y, Liu C, Song W, Wang F, Zhang J, et al: DNA methylation downregulated mir-10b acts as a tumor suppressor in gastric cancer. Gastric Cancer 18: $43-54,2015$.
4. Ng EK, Chong WW, Jin H, Lam EK, Shin VY, Yu J, Poon TC, $\mathrm{Ng}$ SS and Sung JJ: Differential expression of microRNAs in plasma of patients with colorectal cancer: A potential marker for colorectal cancer screening. Gut 58: 1375-1381, 2009.

5. Loffeld RJ, Willems I, Flendrig JA and Arends JW: Helicobacter pylori and gastric carcinoma. Histopathology 17: 537-541, 1990.

6. Recavarren-Arce S, León-Barúa R, Cok J, Berendson R, Gilman RH, Ramírez-Ramos A, Rodríguez C and Spira WM: Helicobacter pylori and progressive gastric pathology that predisposes to gastric cancer. Scand J Gastroenterol Suppl 181: 51-57, 1991.

7. Wang B, Yong H, Zhu H, Ni D, Tang S, Zhang S, Wang W, Zhou Y, Zhao W, Ding G, et al: Abnormal amphiregulin expression correlates with gastric cancer prognosis. Oncotarget 7: 76684-76692, 2016

8. Abu-Farha M, Al-Khairi I, Cherian P, Chandy B, Sriraman D, Alhubail A, Al-Refaei F, AlTerki A and Abubaker J: Increased ANGPTL3, 4 and ANGPTL8/betatrophin expression levels in obesity and T2D. Lipids Health Dis 15: 181, 2016.

9. Masuko K: Angiopoietin-like 4: A molecular link between insulin resistance and rheumatoid arthritis. J Orthop Res 35: 939-943, 2017.

10. Parri M, Pietrovito L, Grandi A, Campagnoli S, De Camilli E, Bianchini F, Marchiò S, Bussolino F, Jin B, Sarmientos P, et al: Angiopoietin-like 7, a novel pro-angiogenetic factor overexpressed in cancer. Angiogenesis 17: 881-896, 2014.

11. Guo L, Li SY, Ji FY, Zhao YF, Zhong Y, Lv XJ, Wu XL and Qian GS: Role of Angpt14 in vascular permeability and inflammation. Inflamm Res 63: 13-22, 2014.

12. Zhu P, Goh YY, Chin HFA, Kersten S and Tan NS: Angiopoietin-like 4: A decade of research. Biosci Rep 32: 211-219, 2012.

13. Tan MJ, Teo Z, Sng MK, Zhu P and Tan NS: Emerging roles of angiopoietin-like 4 in human cancer. Mol Cancer Res 10: 677-688, 2012.

14. Kim SH, Park YY, Kim SW, Lee JS, Wang D and DuBois RN: ANGPTL4 induction by prostaglandin E2 under hypoxic conditions promotes colorectal cancer progression. Cancer Res 71: 7010-7020, 2011.

15. Nakayama T, Hirakawa H, Shibata K, Nazneen A, Abe K, Nagayasu T and Taguchi T: Expression of angiopoietin-like 4 (ANGPTL4) in human colorectal cancer: ANGPTL4 promotes venous invasion and distant metastasis. Oncol Rep 25: 929-935, 2011.

16. Ifon ET, Pang AL, Johnson W, Cashman K, Zimmerman S, Muralidhar S, Chan WY, Casey J and Rosenthal LJ: U94 alters FN1 and ANGPTL4 gene expression and inhibits tumorigenesis of prostate cancer cell line PC3. Cancer Cell Int 5: 19, 2005.

17. Ayaz L, Görür A, Yaroğlu HY, Özcan C and Tamer L: Differential expression of microRNAs in plasma of patients with laryngeal squamous cell carcinoma: Potential early-detection markers for laryngeal squamous cell carcinoma. J Cancer Res Clin Oncol 139: 1499-1506, 2013.

18. Hanahan D and Weinberg RA: Hallmarks of cancer: The next generation. Cell 144: 646-674, 2011.

19. Chiodoni C, Colombo MP and Sangaletti S: Matricellular proteins: From homeostasis to inflammation, cancer, and metastasis. Cancer Metastasis Rev 29: 295-307, 2010.

20. Bornstein P: Matricellular proteins: An overview. J Cell Commun Signal 3: 163-165, 2009

21. Arnold SA and Brekken RA: SPARC: A matricellular regulator of tumorigenesis. J Cell Commun Signal 3: 255-273, 2009.

22. Zhu P, Tan MJ, Huang RL, Tan CK, Chong HC, Pal M, Lam CR, Boukamp P, Pan JY, Tan SH, et al: Angiopoietin-like 4 protein elevates the prosurvival intracellular $\mathrm{O}_{2}(-): \mathrm{H}_{2} \mathrm{O}_{2}$ ratio and confers anoikis resistance to tumors. Cancer Cell 19: 401-415, 2011.

23. Zhang H, Wong CC, WeiH, Gilkes DM,Korangath P, Chaturvedi $\mathrm{P}$, Schito L, Chen J, Krishnamachary B, Winnard PT Jr, et al: HIF-1-dependent expression of angiopoietin-like 4 and L1CAM mediates vascular metastasis of hypoxic breast cancer cells to the lungs. Oncogene 31: 1757-1770, 2012.

24. Nakayama T, Hirakawa H, Shibata K, Abe K, Nagayasu T and Taguchi T: Expression of angiopoietin-like 4 in human gastric cancer: ANGPTL4 promotes venous invasion. Oncol Rep 24: 599-606, 2010

25. Liu X, Yu X, Xie J, Zhan M, Yu Z, Xie L, Zeng H, Zhang F, Chen G, Yi X, et al: ANGPTL2/LILRB2 signaling promotes the propagation of lung cancer cells. Oncotarget 6: 21004-21015, 2015 . 
26. Kikuchi R, Tsuda H, Kozaki K, Kanai Y, Kasamatsu T, Sengoku K, Hirohashi S, Inazawa J and Imoto I: Frequent inactivation of a putative tumor suppressor, angiopoietin-like protein 2, in ovarian cancer. Cancer Res 68: 5067-5075, 2008.

27. Yoshinaga T, Shigemitsu T, Nishimata H, Takei T and Yoshida M Angiopoietin-like protein 2 is a potential biomarker for gastric cancer. Mol Med Rep 11: 2653-2658, 2015.

28. Huang RL, Teo Z, Chong HC, Zhu P, Tan MJ, Tan CK, Lam CR, Sng MK, Leong DT, Tan SM, et al: ANGPTL4 modulates vascular junction integrity by integrin signaling and disruption of intercellular VE-cadherin and claudin-5 clusters. Blood 118: 3990-4002, 2011.

29. Young $\mathrm{K}$ and Chau I: Targeted therapies for advanced oesophagogastric cancer: Recent progress and future directions. Drugs 76: $13-26,2016$
30. Ajani JA: Evolving chemotherapy for advanced gastric cancer. Oncologist 10 (Suppl 3): 49-58, 2005.

31. Van Cutsem E, Dicato M, Geva R, Arber N, Bang Y, Benson A, Cervantes A, Diaz-Rubio E, Ducreux M, Glynne-Jones R, et al: The diagnosis and management of gastric cancer: Expert discussion and recommendations from the 12th ESMO/World Congress on Gastrointestinal Cancer, Barcelona, 2010. Ann Oncol 22 (Suppl 5): v1-v9, 2011.

32. Kostova I: Platinum complexes as anticancer agents. Recent Patents Anticancer Drug Discov 1: 1-22, 2006.

33. Sun Y, Long J and Zhou Y: Angiopoietin-like 4 promotes melanoma cell invasion and survival through aldolase A. Oncol Lett 8: 211-217, 2014. 